\title{
ROTINA COMPUTACIONAL PARA A DETERMINAÇÃO DA VELOCIDADE DE SEDIMENTAÇÃO DAS PARTÍCULAS DO SOLO EM SUSPENSÃO NO ESCOAMENTO SUPERFICIAL ${ }^{1}$
}

\author{
LUIZ F. C. DE OLIVEIRA ${ }^{2}$, MAURO A. MARTINEZ ${ }^{3}$, \\ FERNANDO F. PRUSKI ${ }^{3}$, NORI P. GRIEBELER ${ }^{4}$, GERALDO C. DE OLIVEIRA ${ }^{4}$
}

RESUMO: O presente trabalho teve como objetivo desenvolver uma rotina computacional para a determinação da velocidade de deposição de partículas em suspensão no escoamento superficial, verificar sua aplicação por intermédio de modelo de transporte de sedimentos e comparar os resultados obtidos com dados experimentais. Empregou-se na rotina o processo iterativo de Newton-Rapshon para a solução das equações empregadas na determinação da velocidade de deposição de partículas em suspensão no escoamento superficial, e na solução da equação do transporte de sedimentos empregouse a técnica das diferenças finitas. Essas rotinas foram empregadas na implementação do modelo MTSES (Modelo para Transporte de Solutos no Solo e no Escoamento Superficial). As velocidades de queda das partículas obtidas pela rotina desenvolvida, em média, foram superestimadas, com erro relativo médio de $0,63 \%$, o que possibilitou a utilização da rotina desenvolvida no MTSES. O modelo MTSES superestimou o total de sedimentos transportados pelo escoamento superficial para todas as intensidades de precipitação empregadas neste estudo, com variações porcentuais de 15,6 a 58,3\%.

PALAVRAS-CHAVE: erosão, escoamento superficial, modelagem.

\section{COMPUTATIONAL ROUTINE FOR THE DETERMINATION OF THE SEDIMENTATION VELOCITY OF THE SOIL PARTICLES IN THE DRAIN}

\begin{abstract}
The present work had as objective to develop a computational routine for the determination of the sedimentation velocity in the drain and to verify its application through a model of transport of sediments and to compare the results obtained with experimental data. It was used in the routine the iterative process of Newton-Rapshon for the solution of the equations applied in the determination of the sedimentation velocity in the drain, and on the solution of the transport of sediments equation was applied the technique of the finite differences. Those routines were used in the implementation of the model MTSES (Model for solute transport in the soil and in the drain). The sedimentation velocities obtained by the developed routine were overestimated, with a medium relative error of $0.63 \%$, which allowed the use of the developed routine in MTSES. The MTSES model overestimated the total of sediments transported by the drain, for all the applied precipitation intensities in this study with variations from 15.6 to $58.3 \%$.
\end{abstract}

KEYWORDS: erosion, drain, modeling.

\footnotetext{
${ }^{1}$ Extraído da tese de doutorado do primeiro autor.

${ }^{2}$ Professor da Escola de Agronomia e Engenharia de Alimentos, UFG, Câmpus Samambaia, Caixa Postal 131, Goiânia - GO, lfco@agro.ufg.br

${ }^{3}$ Professor do Departamento de Engenharia de Agrícola, UFV, Viçosa - MG, mmauro@mail.ufv.br

${ }^{4}$ Professor da Escola de Agronomia e Engenharia de Alimentos, UFG, Goiânia - GO, nori@agro.ufg.br

Recebido pelo Conselho Editorial em: 17-10-2003

Aprovado pelo Conselho Editorial em: 19-3-2005
}

Eng. Agríc., Jaboticabal, v.25, n.1, p.126-136, jan./abr. 2005 


\section{INTRODUÇÃO}

O escoamento superficial constitui o principal meio de contaminação dos mananciais de água superficial devido ao transporte de sedimentos e produtos químicos. $\mathrm{O}$ transporte de produtos químicos pelo escoamento superficial pode ter efeito direto e imediato na deterioração da qualidade da água, ao passo que o transporte de material sólido pode ter impacto a longo prazo sobre os recursos hídricos.

A erosão hídrica é o processo de desprendimento e transporte de partículas do solo causado pela água. Constitui-se em uma das principais causas de deterioração acelerada das terras utilizadas na agricultura. Segundo LE BISSONNAIS \& SINGER (1988), a erosão hídrica resulta da interação de forças ativas, como as características da chuva, a declividade do terreno e a capacidade do solo em absorver água, e de forças passivas, como a resistência que o solo exerce à ação erosiva da água, os métodos de cultivo e a densidade da cobertura vegetal. A resistência do solo determina a sua erodibilidade, que é a tendência inerente do solo de erodir-se em diferentes proporções, devido unicamente às diferenças peculiares de cada classe de solo.

A chuva e o escoamento superficial são os agentes energéticos que acarretam a ocorrência da erosão do solo. Essa energia age tanto no sentido de separar as partículas e os agregados do solo, como no transporte desses para o leito dos rios. O material erodido apresenta-se disperso, em suspensão ou na forma de agregados de vários tamanhos, que são transportados pelo escoamento superficial. $\mathrm{O}$ aumento do transporte de partículas e agregados do solo dá-se com o aumento da velocidade e com a espessura da lâmina de água. A velocidade da água, por sua vez, aumenta com o aumento da declividade e com a redução da rugosidade da superfície do solo (PARKER et al., 1995).

Os sedimentos transportados junto à superfície do solo movem-se por salpico, rolagem e arraste e são constituídos de partículas grandes, cujo movimento é altamente dependente do transporte. Um decréscimo na capacidade de transporte causa a imediata deposição dessas partículas. Já os sedimentos em suspensão, os quais estão uniformemente distribuídos na lâmina de água escoada superficialmente, não são depositados imediatamente devido ao decréscimo na capacidade de transporte. Esse retardamento é resultado da pequena velocidade de sedimentação das partículas em suspensão (GRAF, 1971; BORDAS \& SEMMELMANN, 1993).

A erosão hídrica compreende três fases físicas distintas: desagregação, transporte e deposição. A desagregação é o processo de quebra e individualização das partículas da massa de solo, sendo causada, basicamente, pelo impacto direto das gotas da chuva contra a superfície do solo e pelo escoamento superficial. As partículas desagregadas salpicam com as gotículas de água e retornam à superfície, podendo vir a selar os poros superficiais, reduzindo a infiltração de água e, em alguns casos, aumentando a sua resistência à erosão pelas forças coesivas. Na medida em que a intensidade da chuva passa a ser maior que a taxa de infiltração, começa o escoamento superficial, dando início à segunda fase do processo, que é o transporte de sedimentos. Quando a energia do escoamento superficial, que depende do volume e da velocidade da água, não é mais suficiente para transportar o material sólido em suspensão, esse deposita-se, caracterizando, assim, a terceira fase (REICHERT \& CABEDA, 1992; BORDAS \& SEMMELMANN, 1993).

O impacto da gota, que ocorre na fase inicial da erosão hídrica, fornece energia cinética capaz de desestruturar as partículas do solo, mas possui baixa capacidade de transporte de sedimentos. $\mathrm{O}$ montante de solo desestruturado pelo impacto das gotas aumenta com velocidade terminal e tamanho das gotas e com a intensidade da chuva. A segunda fase da erosão hídrica é caracterizada pela remoção de camada superficial do solo. Esse tipo de erosão está associado ao escoamento superficial, que muitas vezes é referenciado como escoamento laminar. Em contraste com o impacto das gotas, o escoamento laminar tem pequena capacidade de desestruturação e alta capacidade de transporte. A erosão em sulcos ocorre quando o escoamento se dá sob forma concentrada e com velocidades altas, 
de modo a vencer as resistências do solo, ou seja, quando a tensão cisalhante associada ao escoamento superficial supera a tensão crítica do solo, que é função das forças coesivas, conduzindo à formação de pequenos canais. As outras formas de erosão representam formas avançadas de erosão por sulcos (BRADFORD et al., 1987; GROSH \& JARRETT, 1994), como as voçorocas.

O desenvolvimento de modelos matemáticos para descrever, com precisão, o transporte de sedimentos pelo escoamento superficial, é bastante difícil, e as equações que descrevem esses processos, em condições reais de campo, apresentam grande complexidade (COX et al., 1994). No entanto, KINOUCHI et al. (1991) e WALLACH \& SHABTAI (1992) consideraram que a evolução dos recursos computacionais, aliada às técnicas numéricas, facilitam a modelagem do transporte de sedimentos, tornando possível integrar os múltiplos processos que determinam as mudanças na concentração dos sedimentos presentes no escoamento superficial no tempo e no espaço, o que pode permitir melhor entendimento do comportamento dos processos associados ao transporte.

Diante do exposto, percebe-se a necessidade do desenvolvimento de modelos computacionais que permitam simular várias situações práticas, que sejam úteis em estudos de estratégias de manejo do solo e preservação ambiental. Nesse sentido, o presente trabalho teve como objetivo desenvolver rotina computacional que permita a determinação da velocidade de deposição das partículas em suspensão no escoamento superficial e verificar sua aplicação por intermédio de modelo de transporte de sedimentos com dados experimentais.

\section{MATERIAL E MÉTODOS}

A rotina computacional para a determinação da velocidade de sedimentação das partículas do solo em suspensão no escoamento superficial foi implementada no modelo MTSES (Modelo para Transporte de Solutos no Solo e no Escoamento Superficial) desenvolvido por OLIVEIRA (1999). O modelo simula o transporte de solutos pelo fluxo de água no perfil do solo e pelo escoamento superficial. No escoamento superficial, o soluto pode ser transportado em solução e adsorvido aos sedimentos extraídos pelo processo erosivo.

Os processos de transporte de solutos são simulados simultaneamente durante um evento chuva pelo MTSES. Na solução da equação de Richards, que rege o fluxo de água no solo (Equação 1), o modelo apresenta a opção de utilizar os modelos de GENUCTHEN (1980) e BROOKS \& COREY (1964) para a determinação da capacidade hídrica específica e condutividade hidráulica do solo nãosaturado em função do potencial matricial [eqs.(2) a (5)].

$$
\begin{aligned}
& \mathrm{C}(\mathrm{h}) \frac{\partial \mathrm{h}}{\partial \mathrm{t}}=\frac{\partial}{\partial \mathrm{z}}\left[\mathrm{K}(\mathrm{h}) \frac{\partial}{\partial \mathrm{z}}(\mathrm{h}-\mathrm{z})\right] \\
& \mathrm{C}(\mathrm{h})=\frac{\mathrm{mn} \alpha^{\mathrm{n}}\left(\theta_{\mathrm{r}}-\theta_{\mathrm{s}}\right) \mathrm{h}^{\mathrm{n}-1}}{\left[1+(\alpha \mathrm{h})^{\mathrm{n}}\right]^{\mathrm{m}+1}} \\
& \mathrm{~K}(\mathrm{~h})=\mathrm{Ko} \mathrm{S}_{\mathrm{e}}{ }^{1 / 2}\left[1-\left(1-\mathrm{S}_{\mathrm{e}}{ }^{1 / \mathrm{m}}\right)^{\mathrm{m}}\right]^{2} \\
& C(h)=\frac{\lambda\left(\theta_{\mathrm{r}}-\theta_{\mathrm{s}}\right)}{\mathrm{h}}\left(\frac{\mathrm{h}_{\mathrm{b}}}{\mathrm{h}}\right)^{\lambda} \\
& \mathrm{K}(\mathrm{h})=\mathrm{K}_{\mathrm{o}}\left(\frac{\mathrm{h}_{\mathrm{b}}}{\mathrm{h}}\right)^{2+3 \lambda}
\end{aligned}
$$

em que, 
$\mathrm{C}(\mathrm{h})$ - capacidade hídrica específica, $\mathrm{L}^{-1}$;

$\mathrm{K}(\mathrm{h})$ - condutividade hidráulica do solo não-saturado, $\mathrm{L} \mathrm{T}^{-1}$;

$\mathrm{K}_{\mathrm{o}}$ - condutividade hidráulica do solo saturado, $\mathrm{L} \mathrm{T}^{-1}$;

$\mathrm{h}$ - potencial matricial, L;

$\mathrm{z}$ - potencial gravitacional, L;

$\mathrm{t}$ - tempo, $\mathrm{T}$;

$S_{\mathrm{e}}$ - grau de saturação efetiva, adimensional, obtido por $S_{\mathrm{e}}=\left[\theta(\mathrm{h})-\theta_{\mathrm{r}}\right] /\left[\theta_{\mathrm{s}}-\theta_{\mathrm{r}}\right]$;

$\theta_{\mathrm{s}}$ e $\theta_{\mathrm{r}}$ - umidades volumétricas de saturação e residual, respectivamente, $\mathrm{L}^{3} \mathrm{~L}^{-3}$;

$\theta(\mathrm{h})$ - umidade volumétrica em função do potencial matricial, $\mathrm{L}^{3} \mathrm{~L}^{-3}$;

$\alpha$ - parâmetro que depende do solo, $\mathrm{L}^{-1}$;

$\mathrm{n}$ - parâmetro que depende do solo, adimensional;

$\mathrm{m}=1-1 / \mathrm{n}$;

$\mathrm{h}_{\mathrm{b}}$ - potencial matricial de entrada de ar, $\mathrm{L}, \mathrm{e}$

$\lambda$ - expoente que depende da distribuição do tamanho de poros, adimensional.

No desenvolvimento do MTSES, foi empregada a equação da conservação da massa de sedimentos proposta por BENNETT (1974), citado por FOSTER (1982) e WICKS \& BATHURST (1996). Para condições de regime variável, a equação do transporte de sedimentos é expressa na forma:

$$
\frac{\partial M_{s}}{\partial x}+\frac{\partial\left(M_{s}\right)}{\partial t}=D_{r}+D_{s}
$$

em que,

$\mathrm{M}_{\mathrm{s}}$ - massa de sedimentos transportada pelo escoamento superficial por unidade de largura e tempo, $\mathrm{M} \mathrm{L}^{-2} \mathrm{~T}^{-1}$;

$\mathrm{x}$ - distância percorrida pelo escoamento superficial, L;

$\mathrm{t}$ - tempo, $\mathrm{T}$;

$\mathrm{D}_{\mathrm{r}}$ - erosão entre sulcos, $\mathrm{M} \mathrm{L}^{-2} \mathrm{~T}^{-1}$, e

$\mathrm{D}_{\mathrm{S}}$ - erosão em sulcos, $\mathrm{M} \mathrm{L}^{-2} \mathrm{~T}^{-1}$.

O primeiro termo do lado esquerdo da igualdade da eq.(6) representa a perda ou o ganho de sedimentos com a distância, e o segundo, a taxa de armazenamento de sedimentos no escoamento.

$\mathrm{O}$ termo $\mathrm{D}_{\mathrm{S}}$ da eq.(1) é diretamente proporcional à diferença entre a capacidade de transporte e à quantidade de sedimentos transportada. FOSTER \& MEYER (1975), citados por FOSTER (1982), SHARMA et al. (1996), COCHRANE \& FLANAGAN (1996) e FRANTI et al. (1996) desenvolveram a eq.(7) para o cálculo de $\mathrm{D}_{\mathrm{S}}$ :

$$
\mathrm{D}_{\mathrm{s}}=\beta\left(\mathrm{T}_{\mathrm{c}}-\mathrm{M}_{\mathrm{s}}\right)
$$

em que,

$\beta$ - coeficiente de reação de primeira ordem para deposição, adimensional, e

$\mathrm{T}_{\mathrm{c}}$ - capacidade de transporte, $\mathrm{M} \mathrm{L}^{-1} \mathrm{~T}^{-1}$.

A eq.(2) introduz o conceito de que a quantidade de sedimentos pode ser diferente da capacidade de transporte. Ocorrerá deposição quando $M_{s}>T_{c}$ e extração quando $M_{s}<T_{c}$ (BORAH \& BORDOLOI, 1989). A capacidade de transporte refere-se à quantidade máxima de sedimentos que o escoamento superficial pode transportar, ou seja, é o balanço entre a taxa de entrada e a de deposição de sedimentos no escoamento.

Com base em estudos efetuados em laboratório para avaliação de deposição de sedimentos, $\beta$ decresce quando: a) o número de Reynolds aumenta, indicando aumento da turbulência do fluxo; 
b) diminui a velocidade de sedimentação de partículas, e c) a vazão aumenta, reduzindo o tempo de sedimentação. Decréscimo de $\beta$ indica que os sedimentos se movimentam por grandes distâncias antes de serem depositados. O coeficiente $\beta$ é obtido pela equação proposta por FOSTER \& MEYER (1975), citados por FOSTER (1982), conforme segue:

$$
\beta=\mathrm{V}_{\mathrm{s}} / 2 \mathrm{Q}
$$

em que,

$\mathrm{V}_{\mathrm{s}}$ - velocidade de sedimentação das partículas em suspensão no escoamento superficial, $\mathrm{L} \mathrm{T}^{-1}$, e $\mathrm{Q}$ - vazão por unidade de largura, $\mathrm{L}^{2} \mathrm{~T}^{-1}$.

Expressão apresentada por GRAF (1971) e BEASLEY \& HUGGINS (1981) foi a utilizada no desenvolvimento do modelo MTSES, para a obtenção da velocidade de queda das partículas em suspensão, conforme segue:

$$
\mathrm{V}_{\mathrm{s}}=\sqrt{\frac{4}{3 \mathrm{C}_{\mathrm{D}}} \mathrm{d}_{\mathrm{p}} \mathrm{g}\left(\rho_{\mathrm{p}}-1\right)}
$$

em que,

$\mathrm{C}_{\mathrm{D}}$ - coeficiente de arrasto, adimensional;

$\mathrm{d}_{\mathrm{p}}$ - diâmetro médio das partículas, $\mathrm{L}, \mathrm{e}$

$\rho_{\mathrm{p}}$ - massa específica da partícula, $\mathrm{M} \mathrm{L}^{-3}$.

O coeficiente de arrasto foi determinado como:

$$
\mathrm{C}_{\mathrm{D}}=\frac{24}{\operatorname{Re}}+\frac{3}{\sqrt{\operatorname{Re}}}+0,34
$$

em que, o número de Reynolds (Re) é dado pela eq.(11):

$$
\mathrm{Re}=\frac{\mathrm{V}_{\mathrm{s}} \mathrm{d}_{\mathrm{p}}}{\mathrm{v}}
$$

em que, $v$ é a viscosidade cinemática da água, $\mathrm{M}^{2} \mathrm{~T}^{-1}$.

Como a velocidade de sedimentação das partículas depende do coeficiente de arrasto e esse, por sua vez, depende da $\mathrm{V}_{\mathrm{s}}$, seu valor é calculado pelo emprego de processo iterativo. Empregou-se na implementação do modelo MTSES, o método de Newton-Rapshon para a solução das eqs.(9), (10) e (11), conforme segue:

Fazendo $K=\frac{4}{3} d_{p} g\left(\rho_{p}-1\right)$, e substituindo as eqs. (10) e (11) na eq.(9), têm-se que:

$$
V_{s}=\sqrt{\frac{24 v}{\frac{d_{p} V_{s}}{\sqrt{\frac{9 v}{d_{p} V_{s}}}+0,34}}}
$$

Escrevendo a eq.(12) como função de $\mathrm{V}_{\mathrm{s}}$ e fazendo sua derivada, tem-se:

$$
\begin{aligned}
& f\left(V_{s}\right)=V_{s}^{2}-F\left(V_{s}\right) \\
& f^{\prime}\left(V_{s}\right)=2 V_{s}-F^{\prime}\left(V_{s}\right)
\end{aligned}
$$

em que,

$$
\mathrm{F}\left(\mathrm{V}_{\mathrm{s}}\right)=\frac{\mathrm{K}}{\mathrm{C}} \quad \text { e } \quad \mathrm{F}^{\prime}\left(\mathrm{V}_{\mathrm{s}}\right)=\frac{\mathrm{C}+\mathrm{KD}}{\mathrm{C}^{2}}
$$




$$
\begin{aligned}
& \mathrm{C}=\frac{\mathrm{A}}{\mathrm{V}_{\mathrm{s}}}+\frac{\mathrm{B}}{\sqrt{\mathrm{V}_{\mathrm{s}}}}+0,34 \text { e } \mathrm{D}=\frac{\mathrm{A}}{\mathrm{V}_{\mathrm{s}}^{2}}+\frac{\mathrm{B}}{\sqrt{4 \mathrm{~V}_{\mathrm{s}}^{3}}} \\
& \mathrm{~A}=24 v / \mathrm{d}_{\mathrm{p}} \text { e } \quad \mathrm{B}=\sqrt{9 v / \mathrm{d}_{\mathrm{p}}}
\end{aligned}
$$

As eqs. (12) a (17) compreendem um sistema de equações não-lineares que serão resolvidas pelo método iterativo de Newton-Rapshon, descrito por OLIVEIRA (1997), conforme segue:

$$
\begin{aligned}
& \mathrm{f}\left(\mathrm{V}_{\mathrm{s}}\right)+\mathrm{f}^{\prime}\left(\mathrm{V}_{\mathrm{s}}\right) \delta \mathrm{V}_{\mathrm{s}}=0 \\
& \delta \mathrm{V}_{\mathrm{s}}=\mathrm{V}_{\mathrm{s}}{ }^{\mathrm{i}+1}-\mathrm{V}_{\mathrm{s}}{ }^{\mathrm{i}}
\end{aligned}
$$

em que, $\delta \mathrm{V}_{\mathrm{s}}$ é a diferença entre os valores de $\mathrm{V}_{\mathrm{s}}$ para i+1 e i, sendo i o número de iterações.

$\mathrm{O}$ processo iterativo é interrompido quando $\delta \mathrm{V}_{\mathrm{s}}$ for menor que o valor preestabelecido na execução da rotina. Esse valor é uma informação de entrada no modelo MTSES, bem como o valor inicial de $\mathrm{V}_{\mathrm{s}}$. No modelo desenvolvido, o "default" do critério de parada $\left(\mathrm{C}_{\mathrm{p}}\right)$ para o processo iterativo foi estabelecido em $10^{-5}$.

Na Figura 1, apresenta-se o fluxograma da rotina para o cálculo da velocidade de sedimentação das partículas em suspensão no escoamento superficial, implementada no modelo MTSES. Para a execução dessa rotina, foi desenvolvido um programa em linguagem Delphi, versão 3.0, chamado VELOQUEDA.

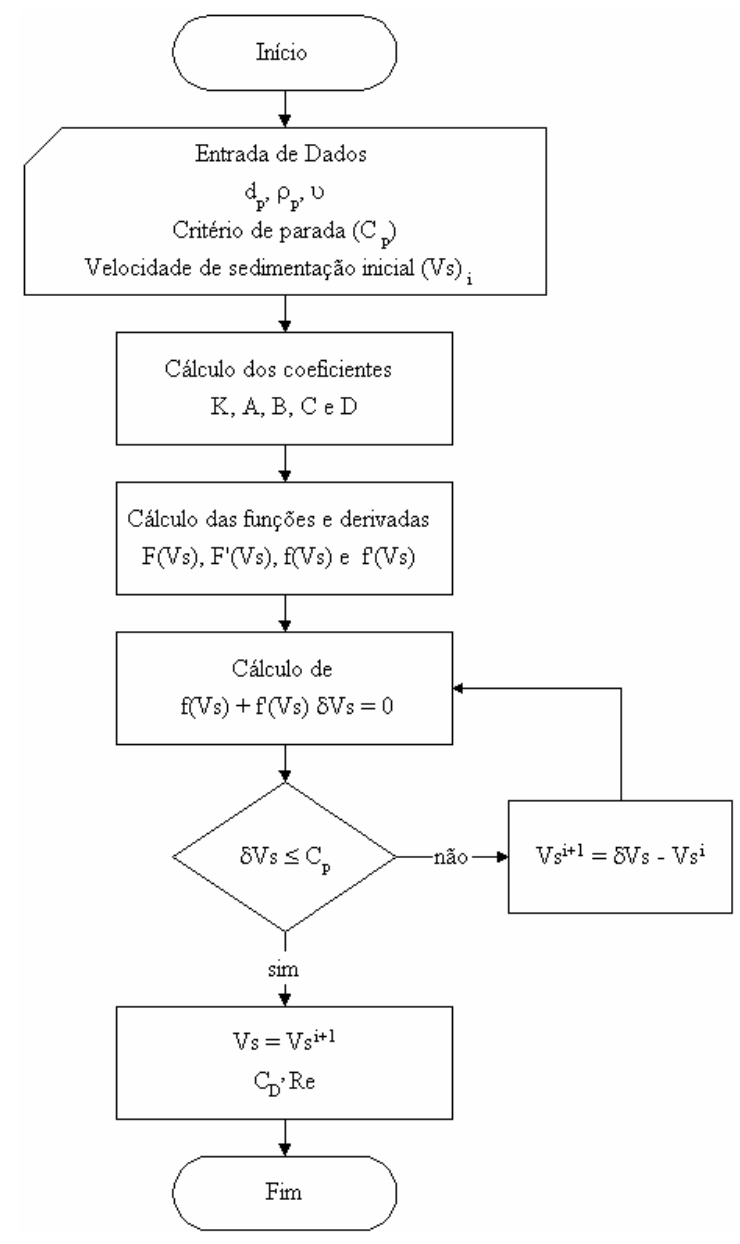

FIGURA 1. Fluxograma da rotina para o cálculo da velocidade de sedimentação das partículas em suspensão no escoamento superficial. 
Para verificar a adequação da rotina desenvolvida, utilizou-se da distribuição granulométrica do solo e as massas específicas das partículas obtidas por BEASLEY \& HUGGINS (1981), conforme apresentado na Tabela 1. Os resultados foram comparados por meio do erro relativo médio que, segundo ALEXANDER \& SKAGGS (1987), é obtido pela seguinte equação:

$$
\mathrm{ERM}=\frac{\sum_{\mathrm{i}=1}^{\mathrm{N}_{\mathrm{OBS}}}\left|\frac{\mathrm{OBS}_{\mathrm{i}}-\mathrm{EST}_{\mathrm{i}}}{\mathrm{OBS}_{\mathrm{i}}}\right|}{\mathrm{N}_{\mathrm{OBS}}}
$$

em que,

OBS e EST - valores observados e estimados da variável de interesse, respectivamente, e

$\mathrm{N}_{\mathrm{OBS}}$ - número de observações.

TABELA 1. Distribuição granulométrica do solo e as massas específicas das partículas.

\begin{tabular}{|c|c|c|c|c|}
\hline \multirow{2}{*}{ Classe } & \multicolumn{3}{|c|}{ Granulometria } & \multirow{2}{*}{$\begin{array}{l}\text { Massa Específica da Partícula } \\
\qquad\left(\mathrm{kg} \mathrm{m}^{-3}\right)\end{array}$} \\
\hline & $\%$ & Fração & Diâmetro $\left(10^{-3} \mathrm{~m}\right)$ & \\
\hline 1 & 10 & Argila & 0,002 & 2.650 \\
\hline 2 & 18 & Silte & 0,010 & 2.650 \\
\hline 3 & 50 & Agregado pequeno & 0,030 & 1.800 \\
\hline 4 & 17 & Agregado grande & 0,500 & 1.600 \\
\hline 5 & 5 & Areia & 0,200 & 2.650 \\
\hline
\end{tabular}

Fonte: BEASLEY \& HUGGINS (1981)

\section{RESULTADOS E DISCUSSÃO}

Com base nos valores apresentados na Tabela 1, calculou-se, para cada classe de partícula do solo, a velocidade de queda das partículas, considerando a viscosidade cinemática da água igual a $10^{-6} \mathrm{~m}^{2} \mathrm{~s}^{-1}$ e o critério de parada do processo iterativo de $10^{-7}$.

Na Tabela 2, apresentam-se os valores das velocidades de queda das partículas obtidas por BEASLEY \& HUGGINS (1981) e calculadas pela rotina VELOQUEDA, os erros relativos (ERM), os coeficientes de arrasto e os números de Reynolds. Em média, o modelo superestimou os valores das velocidades de queda das partículas, com erro relativo médio (ERM) de 0,63\%, o que possibilita a utilização da rotina desenvolvida em modelos de simulação do transporte de sedimentos. O maior desvio $(5,4 \%)$ foi verificado na classe 1 referente à fração argila, para a qual não se tem a correta definição do tamanho dessa partícula.

TABELA 2. Velocidade de queda das partículas obtidas por BEASLEY \& HUGGINS (1981) e pela rotina VELOQUEDA**

\begin{tabular}{|c|c|c|c|c|c|}
\hline \multirow{2}{*}{ Classe } & $\mathrm{V}_{\mathrm{SBH}}{ }^{*}$ & $\mathrm{~V}_{\mathrm{S} \text { VELOQUEDA }}{ }^{* *}$ & \multirow{2}{*}{$\begin{array}{c}\text { ERM } \\
\%\end{array}$} & \multirow{2}{*}{$\mathrm{C}_{\mathrm{D}}$} & \multirow{2}{*}{$\operatorname{Re}$} \\
\hline & \multicolumn{2}{|c|}{$\mathrm{m} \mathrm{s}^{-1}$} & & & \\
\hline 1 & 0,0000036000 & 0,0000037931 & 5,40 & $3,00 \times 10^{6}$ & $7,59 \times 10^{-6}$ \\
\hline 2 & 0,0000896000 & 0,0000895135 & 0,10 & $2,69 \times 10^{4}$ & $8,94 \times 10^{-4}$ \\
\hline 3 & 0,0003910000 & 0,0003870724 & 1,00 & $2,10 \times 10^{3}$ & $1,16 \times 10^{-2}$ \\
\hline 4 & 0,4320820000 & 0,4329408574 & 0,20 & 0,66 & $2,16 \times 10^{2}$ \\
\hline 5 & 0,0263375000 & 0,0259933967 & 1,31 & 6,27 & 5,20 \\
\hline Média & & & 0,63 & & \\
\hline
\end{tabular}


$\mathrm{Na}$ Figura 2, apresentam-se as curvas da quantidade de sedimentos transportados pelo escoamento superficial obtida experimentalmente por OLIVEIRA (1999) e calculada pelo modelo MTSES, empregando a eq.(1) para três intensidades de precipitação $\left(66,6 ; 82,4\right.$ e $\left.100,4 \mathrm{~mm} \mathrm{~h}^{-1}\right) \mathrm{e}$ para os incremento na distância percorrida pelo escoamento superficial e tempo de $0,10 \mathrm{~m}$ e 30 segundos, respectivamente. $\mathrm{O}$ diâmetro médio de partículas considerado nas simulações foi o equivalente à fração argila de $0,002 \mathrm{~mm}$, uma vez que os sedimentos coletados no teste de campo foram constituídos de argila dispersa em água.

Pode-se observar na Figura 2 que, para os 600; 840 e 900 segundos iniciais, houve maior incremento na taxa de extração de sedimentos para as intensidades de precipitação de 100,4; 82,4 e $66,6 \mathrm{~mm} \mathrm{~h}^{-1}$, respectivamente. Esse comportamento deve-se ao fato de que, no início do escoamento superficial, houve transporte das partículas de solo soltas sobre a superfície do solo e liberadas pelo impacto das gotas da chuva. Após esses períodos iniciais, observou-se redução no incremento na taxa de transporte de sedimentos, provavelmente acarretada pelo selamento superficial provocado pelo impacto das gotas das chuvas, que promoveu o aumento das forças coesivas, principalmente para as maiores intensidades de precipitação.

$\mathrm{Na}$ Tabela 3, estão apresentados os totais de sedimentos transportados pelo escoamento superficial. O total de sedimentos obtidos nas simulações apresentou os menores desvios em relação ao observado quando se empregou o modelo de Brooks e Corey na solução da equação de Richards com a finalidade de determinar a infiltração da água no solo, por subestimar os valores de vazão, reduzindo, assim, a capacidade de transporte.

Embora os valores de ERM sejam altos, a variação da taxa de sedimentos transportada pelo escoamento superficial, obtida por simulação, apresentou comportamento semelhante ao observado no campo, com aumento dos sedimentos transportados com a elevação da vazão escoada superficialmente, o que não invalida a rotina desenvolvida para simular a velocidade de sedimentação das partículas do solo em suspensão no escoamento superficial. Esse comportamento deve-se ao aumento da capacidade de transporte com o incremento da vazão, propiciando a extração do solo e reduzindo a velocidade de deposição de sedimentos.

TABELA 3. Total de sedimentos transportados pelo escoamento superficial, observados e simulados, usando o MTSES e os modelos de Genuchten (VG) e Brooks \& Corey (BC).

\begin{tabular}{cccccc}
\hline Intensidade de & \multicolumn{5}{c}{ Total de Sedimentos $(\mathrm{kg})$} \\
\cline { 2 - 6 } Precipitação $\left(\mathrm{mm} \mathrm{h}^{-1}\right)$ & OBS & VG & ERM $(\%)$ & BC & ERM (\%) \\
\hline 100,4 & 0,5916 & 0,9367 & 58,3 & 0,8915 & 50,7 \\
82,4 & 0,4445 & 0,5519 & 24,2 & 0,5395 & 21,4 \\
66,6 & 0,4345 & 0,5258 & 21,0 & 0,5024 & 15,6 \\
\hline
\end{tabular}


(a)



(b)

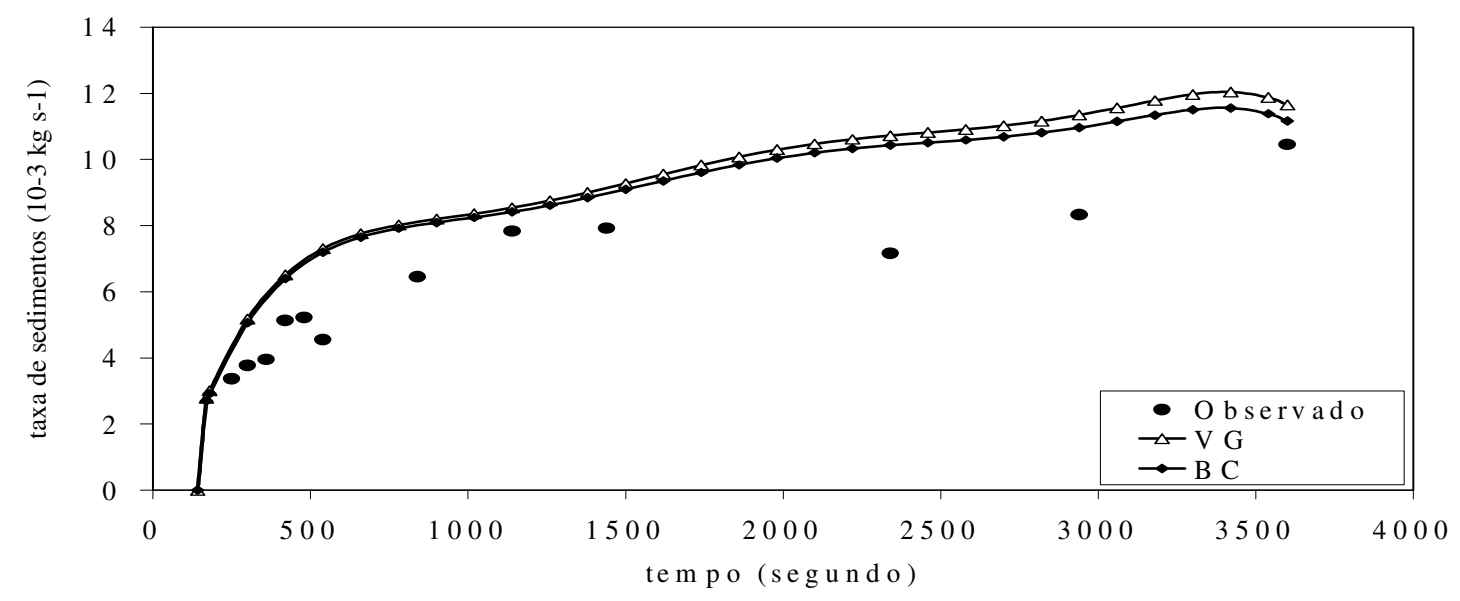

(c)

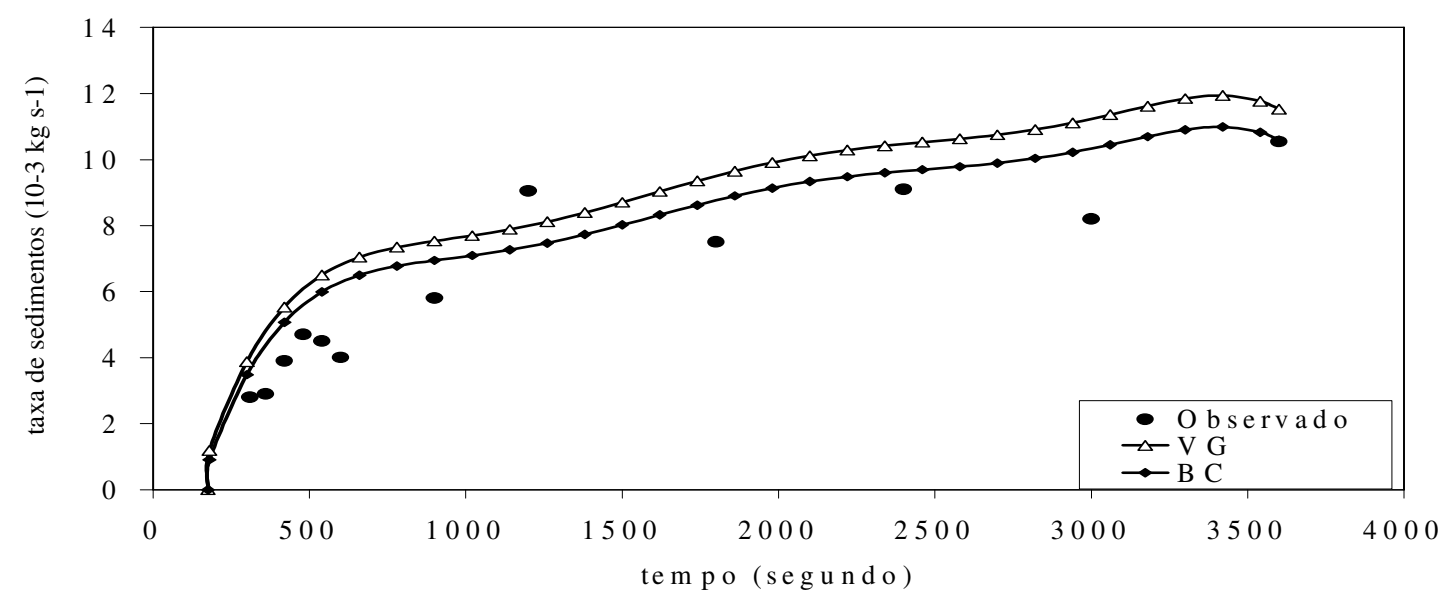

FIGURA 2. Sedimentos transportados pelo escoamento superficial, observados e simulados pelo modelo MTSES empregando os modelos de Genuchten (VG) e Brooks \& Corey (BC), para as intensidades de precipitação de (a) $100,4 \mathrm{~mm} \mathrm{~h}^{-1}$, (b) $82,4 \mathrm{~mm} \mathrm{~h}^{-1}$ e (c) $66,6 \mathrm{~mm} \mathrm{~h}^{-1}$. 


\section{CONCLUSÕES}

A rotina VELOQUEDA superestima, em média, as velocidades de queda das partículas nos padrões testados; contudo, com erro relativo médio de $0,63 \%$, indica ser possível utilizar a rotina desenvolvida no modelo para transporte de solutos no solo e no escoamento superficial (MTSES). O modelo MTSES superestima o total de sedimentos transportados pelo escoamento superficial para todas as intensidades de precipitação empregadas neste estudo, com variações porcentuais, para o erro relativo médio, entre 15,6 a $58,3 \%$.

\section{REFERÊNCIAS}

ALEXANDER, L.; SKAGGS, R.W. Predicting unsaturated hydraulic conductivity from the soil texture. Journal of Irrigation and Drainage Engineering, New York, v.113, n.2, p.184-97, 1987.

BEASLEY, D.B.; HUGGINS L. F. Answers: User's manual. Chicago: United States Environmental Protection Agency, 1981. 51 p.

BORAH, D.K.; BORDOLOI, P. K. Nonuniform sediment transport model. Transactions of the ASAE, St. Joseph, v.32, n.5, p.1631-6, 1989.

BORDAS, M.P.; SEMMELMANN, F.R. Elementos de engenharia de sedimentos. In: TUCCI, C.E.M. Hidrologia: ciência e aplicação. Porto Alegre: UFGS; São Paulo: USP/ABRH, 1993. p.915-43.

BRADFORD, J.M.; FERRIS, J.E.; REMLEY, P.A. Interril soil processes: I. Effect of surface seling on infiltration, runoff and soil splash detachment. Soil Science Society of America, Madison, v.51, n.6, p.1566-71, 1987.

BROOKS, R.H.; COREY, A.T. Hydraulic properties of porous media. Fort Collins: Colorado State University, 1964. $27 \mathrm{p}$.

COX, C. L.; JONES, W.F.; QUISENBERRY, V.L.; YO, F. One-dimensional infiltration with moving finite elements and improved soil water diffusivity. Water Resources Research, Washington, v.30, n.5, p.1431-8, 1994.

COCHRANE, T.A.; FLANAGAN, D.C. Detachment in a simulated rill. Transactions of the ASAE, St. Joseph, v.40, n.1, p.111-19, 1996.

FOSTER, G.R. Modeling the erosion process. In: HAAN, C.T.; JOHNSON, H.P.; BRAKENSIEK, D.L. Hydrologic modeling of small watersheds. St. Joseph: ASAE, 1982. p.297-380.

FRANTI, T.G.; FOSTER, G.R.; MONKE, E.J. Modelling the effects of incorporater residue on rill erosion - Part I: Model development and sensitivity analysis. Transactions of the ASAE, St. Joseph, v.39, n.2, p.535-5, 1996.

GENUCHTEN, M.T. van. A closed-form equation for predicting the hydraulic conductivity of unsaturated soils. Soil Science Society America Journal, Madison, v.44, p.892-8, 1980.

GRAF, W.H. Hydraulics of sediment transport. New York: McGraw-Hill, 1971. 509 p.

GROSH, J.L.; JARRETT, A.R. Interril erosion and runoff steep slopes. Transactions of the ASAE, St. Joseph, v.37, n.4, p.1127-33,1994.

KINOUCHI, T.; KANDA, M.; HINO, M. Numerical simulation of infiltration and solute transport in S-shaped model basin by a boundary-fitted grid system. Journal of Hydrology, Amsterdam, v.122, n.1/4, p.373-406,1991.

LE BISSONNAIS, Y.; SINGER, M.J. Seal formation, runoff, and interill erosion from seveteen California soils. Soil Science Society of America, Cincinnati, v.57, n.3, p.781-4, 1988. 
OLIVEIRA, L.F.C. Modelo para transporte de solutos no solo e no escoamento superficial. 1999. 171 f. Tese (Doutorado em Irrigação e Drenagem) - Universidade Federal de Viçosa, Viçosa - MG, 1999.

OLIVEIRA, L.F.C. Dimensionamento de linhas laterais de irrigação utilizando o método iterativo de Newton-Rapshon. Engenharia Rural, Piracicaba, v.8, n.1, p.81-90, 1997.

PARKER, D.B.; MICHEL, T.G.; SMITH, J.L. Compaction and water velocity effects on soil in shallow flow. Journal of Irrigation and Drainage Engineering, New York, v.121, n.2, p.170-8, 1995.

REICHERT, J.M.; CABEDA, M.S. Salpico de partículas e escoamento superficial. Revista Brasileira de Ciência do Solo, Campinas, v.16, n.3, p.390-6, 1992.

SHARMA, K.D.; MURTHY, J.S.R.; DHIR, R.P. Modelling sediment delivery in an arid upland basins. Transactions of the ASAE, St. Joseph, v.39, n.2, p.517-24, 1996.

WALLACH, R.; SHABTAI, R. Modeling surface runoff contamination by chemicals under transient water infiltration. Journal of Hydrology, Amsterdam, v.132, n.1/4, p.263-81, 1992.

WICKS, J.M.; BATHURST, J.C.; SHESED, A. A physically based, distributed erosion and sediment yield component for the hydrological modelling system. Journal of Hydrology, Amsterdam, v.175, n.1/4. p.213-38,1996. 